\title{
Composition of high frequency ambient noise from cross-correlation: A case study using a small aperture array*
}

\author{
Weitao Wang ${ }^{1, \star}{\text { Sidao } \mathrm{Ni}^{2} \text { and Baoshan Wang }}^{1}$ \\ ${ }^{1}$ Institute of Geophysics, China Earthquake Administration, Beijing 100081, China \\ ${ }^{2}$ Mengcheng National Geophysical Observatory, School of Earth and Space Science, \\ University of Science and Technology of China, Hefei 230026, China
}

\begin{abstract}
Long-time cross correlation of ambient noise has been proved as a powerful tool to extract Green's function between two receivers. The study of composition of ambient noise is important for a better understanding of this method. Previous studies confirm that ambient noise in the long period ( $3 \mathrm{~s}$ and longer) mostly consists of surface wave, and $0.25-2.5$ $\mathrm{s}$ noise consists more of body waves. In this paper, we perform cross correlation processing at much higher frequency (30-70 $\mathrm{Hz}$ ) using ambient noise recorded by a small aperture array. No surface waves emerge from noise correlation function (NCF), but weak $\mathrm{P}$ waves emerge. The absence of surface wave in NCF is not due to high attenuation since surface waves are strong from active source, therefore probably the high ambient noise mostly consists of body wave and lacks surface wave. Origin of such high frequency body waves in ambient noise remains to be studied.
\end{abstract}

Key words: ambient noise; cross correlation; Green's function; body wave; high frequency

CLC number: P315.3 Document code: A

\section{Introduction}

Extraction of empirical Green's function from long-time cross correlation of ambient noise is a rapidly emerging field in recent seismology research. It is shown that the cross-correlation of the ambient noise field will yield the Green's function between the two receivers, as if one of the receivers acts as an impulse source (Weaver and Lobkis, 2001, 2004; Derode et al., 2003; Snieder, 2004; Larose et al., 2005). Application of this method to large amount of continuous digital seismic records provides us a new approach for imaging the Earth. Surface wave tomography using Green's function calculated from ambient noise has been used to study the crust and upper mantle structure within the Earth, from local scales to continental scales (Sabra et al., 2005; Shapiro et al., 2005; Yao et al., 2006; Lin et al., 2007; Yang et al., 2007; Bensen et al., 2008; Zheng et al.,

\footnotetext{
* Received 14 May 2010; accepted in revised form 5 August 2010; published 10 October 2010 .

^ Corresponding author. e-mail: wangwt@cea-igp.ac.cn

(C) The Seismological Society of China and Springer-Verlag Berlin Heidelberg 2010
}

2008). Some authors claim that the whole process of cross correlation between two receivers is repeatable, thus providing us a tool to monitor temporal variation of seismic wave velocity of the medium by tracking the time shift of the Green's function. Such technique has been used to monitoring velocity change in fault zones and volcanoes (Brenguier et al., 2008a, b; Duputel et al., 2009; Xu and Song, 2009).

When receivers are widely separated, the coherent propagation noise must have sufficient amplitude to be recorded by both receivers. Fundamental surface wave has dominated the Green's function obtained from cross correlation of ambient noise due to its low attenuation as well as geometrical spreading. Wapenaar (2004) claimed that both surface wave and body wave can be retrieved by cross correlation. This is confirmed by recent studies which demonstrate the presence of $\mathrm{P}$ wave in noise correlation functions (NCFs) (Roux et al., 2005; Draganov et al., 2007; Landès et al., 2010). Zhan et al. (2010) reported that Moho post-critical body waves ( $\mathrm{SmS}$ and its multiples) can be retrieved from NCF in the period band of $1-5 \mathrm{~s}$, they also recognized that $\mathrm{SmS}$ can be retrieved 
only for stable continents where Moho is flat. Therefore, body waves from NCF are still relatively rare cases, and this demands for more studies of body waves from ambient noises.

A better understanding of the composition and origin of ambient noise is important to improve the accuracy of noise based tomography and monitoring. It is well known that ambient noise is not flat in frequency domain. Two strong peaks of seismic noise are topically observed in the primary (around $15 \mathrm{~s}$ period) and secondary periods (around $7.5 \mathrm{~s}$ period). The origin of primary and secondary microseisms has not been well understood; the primary microseism is believed to be related to direct forcing of strong oceanic waves (Hasselmann, 1963). The secondary microseism is generated by the nonlinear interaction between two primary waves with the same frequency and opposite direction (Longuet-Higgins, 1950). The exact source location of secondary microseism is still under debate (Rhie and Romanowicz, 2006; Stehly et al., 2006; Kedar et al., 2008; Yang and Ritzwoller, 2008). At longer period, seismic noise with period above $100 \mathrm{~s}$ is observed and named as Earth hum, which is believed to be related to the ocean infragravity wave (Rhie and Romanowicz, 2004, 2006). It is well accepted that in classic microseism band 5-20 s seismic noise filed predominantly consists of fundamental mode of Rayleigh and Love waves. In short period, the body wave plays an important role because in such a period high-mode surface wave rapidly attenuates with distance. Koper et al. (2010) made a global survey of noise field in 0.25-2.5 s and found that body waves, such as $\mathrm{Lg}, \mathrm{P}$ waves, constitute most of ambient noise at these periods.

Most of previous studies consider ambient noise with frequency below $5 \mathrm{~Hz}$, partly due to the large distance between stations they used. In this paper, we demonstrate the result of long-time cross correlation in high frequency $(>10 \mathrm{~Hz})$, using three weeks long records from a small aperture array deployed in Sichuan province of China. Our result shows that weak $\mathrm{P}$ wave instead of surface wave shows up in the NCFs, indicating that probably the high frequency noise mostly consists of body wave and lacks surface wave.

\section{Data set}

After Wenchuan $M_{\mathrm{S}} 8.0$ earthquake we deployed a small aperture array at the northeast edge of the main fault. The array consists of eight receivers, each of which uses Guralp 40T as sensor and GPS synchronized Reftek $130 \mathrm{~B}$ as data acquisition system. These receivers were deployed as a cross with inter-receiver distance about 80-100 m (Figure 1). All these receivers started acquisition from June 62008 to June 292008 with sampling frequency $500 \mathrm{~Hz}$. The three weeks high sampling rate continuous records provided us a good opportunity to study the property of high frequency ambient noise cross correlation with sufficient short inter-receiver distance.

Furthermore, to monitor the state of stress in this area, we also conducted an active source experiment during this period. We used an electric hammer as seismic source to monitor the velocity change, which is shown as filled square in Figure 1. A receiver which is labeled $O$ is placed just near the active source to record the near-source signal. The body wave and surface wave excited by this active source were well recorded by all these receivers, which thus makes it possible to perform further comparison and phase identification of noise cross correlation functions. Moreover, a surface wave inversion by refraction common mid-point imaging was performed, which provided us a primary understanding of subsurface velocity structure (Huang et al., 2009).

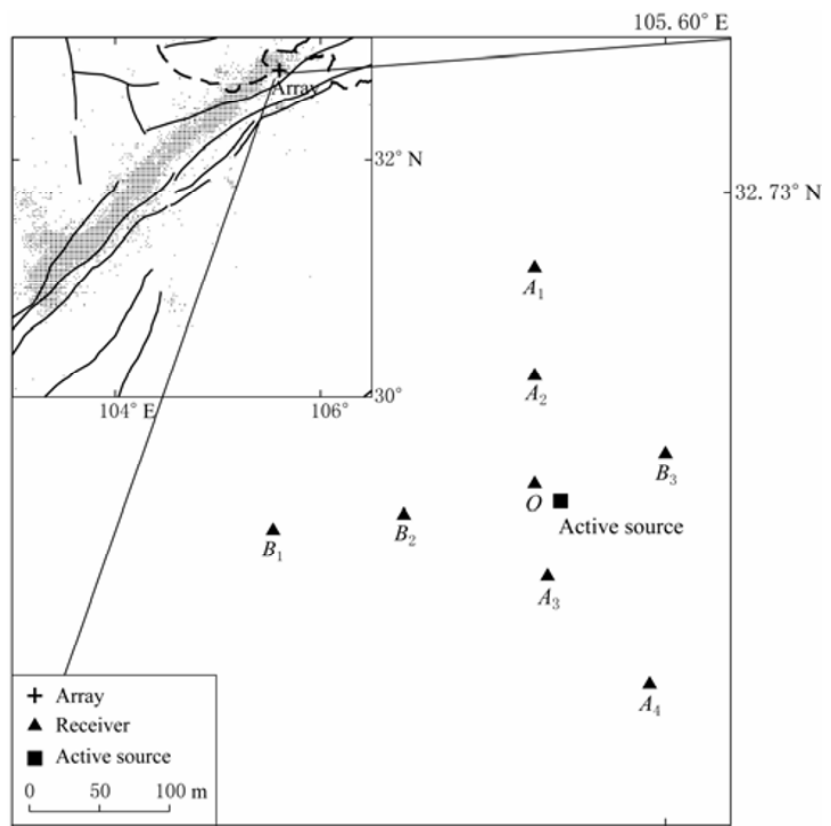

Figure 1 Position and layout of the small aperture array used in this study. Small cross indicates the array's position with respect to Longmenshan fault.

\section{Data processing and results}

The vertical components of records were used to obtain the cross correlation function. We prepared daily 
records for each receiver, and then removed the instrument response. The strong signal excited by active source was recorded by these receivers. In order to reduce the contamination by these signals, we set the amplitude of records to zero when our active source was working. Since the active source only worked during the first ten minutes of each hour, this does not affect the whole ambient noise records used for later cross correlation processing.

For our experiment was conducted after Wenchuan $M_{\mathrm{S}} 8.0$ earthquake, many aftershocks were recorded by these receivers. Following Bensen et al. (2007), we used one-bit method to normalize each record in time domain then equalized the noise spectrum from $2 \mathrm{~s}$ to $100 \mathrm{~Hz}$.

Figure 2 displays the spectrum of station $A_{1}$ before and after one bit normalization are applied. Spectral peaks are kept after the normalization process, and only the relative strengths of spectra peaks (around 10-20 Hz, and $30-70 \mathrm{~Hz}$ ) are different before and after the normalization. Since there is a peak between $30 \mathrm{~Hz}$ and 70 $\mathrm{Hz}$, it is probable that the energy in this frequency band leads to emergence of coherent arrivals on NCFs.
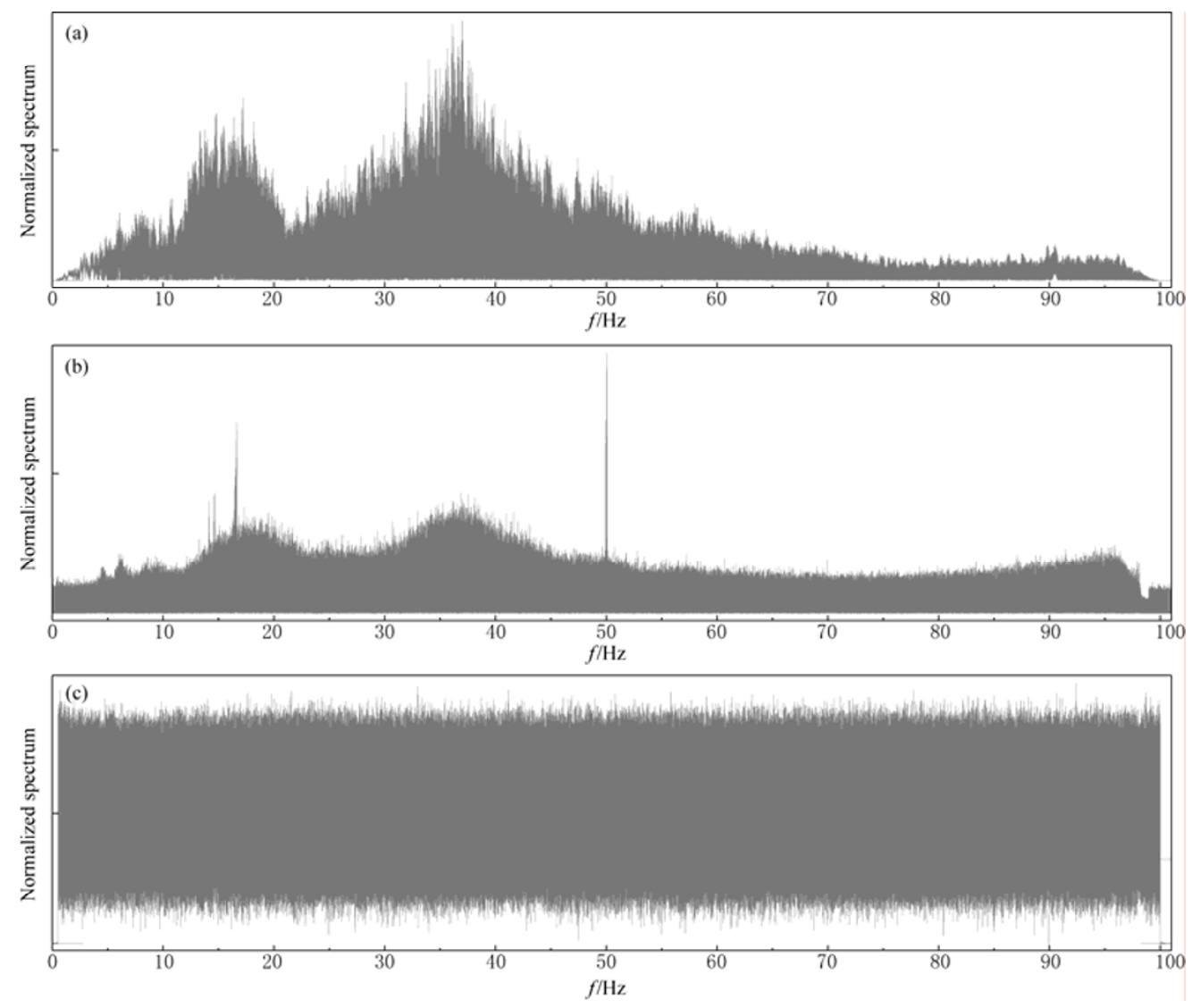

Figure 2 Normalized spectrum of noise records at receiver $A_{1}$. (a) Power spectrum of noise before time domain one bit clip. (b) Power spectrum of noise after one bit clip. (c) Power spectrum of noise after one bit clip and $2 \mathrm{~s}$ to $100 \mathrm{~Hz}$ spectrum whitening. Each spectrum is normalized by its maximum.

We applied frequency whitening after one bit normalization in order to broaden the frequency band in the cross correlation function. After time normalization and spectrum whitening, we calculated the cross correlation functions between receiver $O$ and other receivers day by day, and then stacked them together to correspond to longer time series. The same procedure was performed for receivers $A_{4}$ and $B_{1}$ which are located on the edge of each line in the array cross.
The stacked NCFs band passed from $30 \mathrm{~Hz}$ to 70 $\mathrm{Hz}$ are plotted as black traces in Figure 3, arranged in increasing inter-receiver distance. The labels on the left of the figure indicate the receiver pairs against which the NCF is calculated. The records of $\mathrm{P}$ wave excited by the active source are overlaid as red traces. Those NCFs whose inter-receiver distances are almost same are not shown to avoid overlapping traces. According to Huang et al. (2009), the surface wave velocity in this area is 
about $300 \mathrm{~m} / \mathrm{s}$, which is plotted as green dashed lines in Figure 3. There seems to be no strong surface wave signals along this line in the NCFs. Also, we estimate the apparent velocity of $\mathrm{P}$ wave using the $\mathrm{P}$ wave records of active source, and the velocity is close to $2 \mathrm{~km} / \mathrm{s}$ (blue dashed line). Along the blue line, we can see some signals in most NCFs, which may be weak $P$ waves extracted from cross correlation of ambient noise. The slope of $2 \mathrm{~km} / \mathrm{s}$ does not fit the data perfect, which may be caused by the path effects of receiver pairs, since not all the receiver pairs we used are in the same direction. Our result shows weak $\mathrm{P}$ wave emerges in the NCFs without obvious surface waves. This indicates, at least in frequency $30-70 \mathrm{~Hz}$, the noise field consists of more body waves and less surface waves.

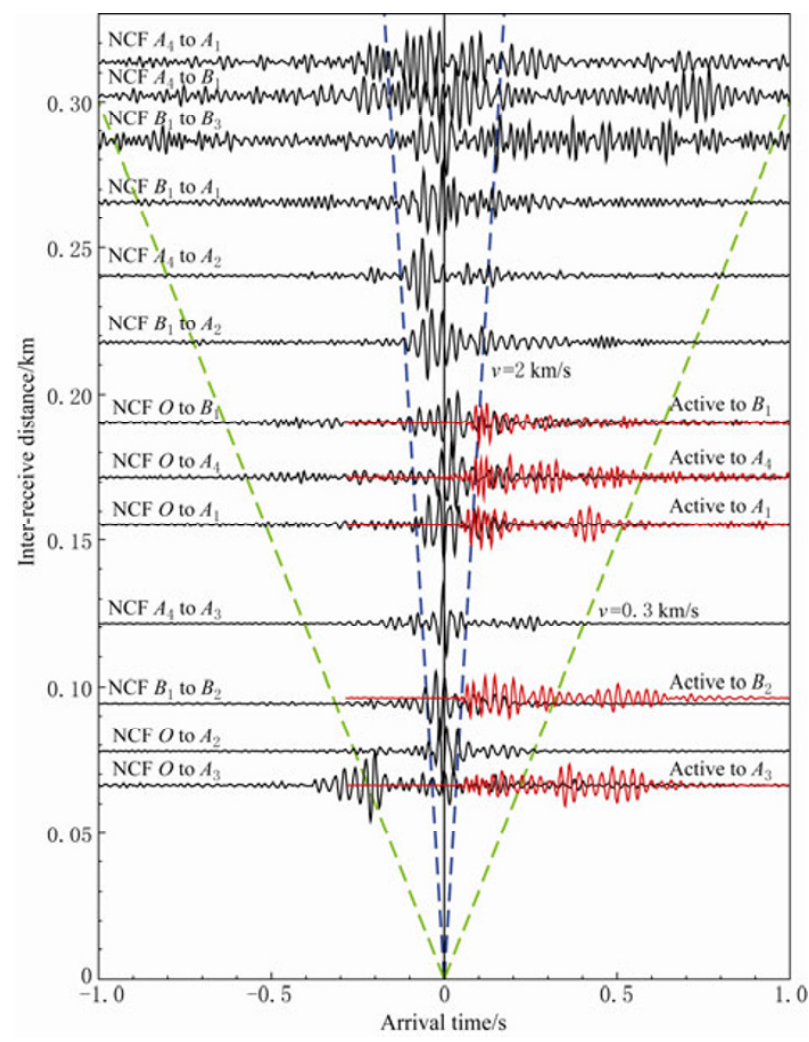

Figure 3 Noise correlation functions from cross correlation of vertical component of each receiver. NCFs are shown as black waveforms with respect to inter-receiver distance together with $\mathrm{P}$ wave excited by active source shown as red waveforms, both are filtered from $30 \mathrm{~Hz}$ to $70 \mathrm{~Hz}$. Labels indicating receiver pair used for each cross correlation are shown on the left above each NCF waveform, the active source and receiver pairs are also labeled on the right above each red waveform. Waves propagating with apparent velocity $2 \mathrm{~km} / \mathrm{s}$ and $300 \mathrm{~m} / \mathrm{s}$ are plotted as blue dashed line and green dashed line separately.

\section{Discussion and conclusions}

Reconstruction of Green's function from ambient noise cross correlation has been extensively studied recently, and the inter-station distance of these works vary from several kilometers to thousands of kilometers. Green's function retrieved from long distance mostly consists of low frequency signals since high frequency noise attenuates quickly with distance. Our experiment provides a good opportunity for studying high frequency noise correlation. But as shown in Figure 3, there exists large amplitude signals around zero lag in the NCFs. This may due to the short inter-station distance of our array. The receivers are extremely close that the largest distance is shorter than $500 \mathrm{~m}$. The local noises, such as wind flow, human activity, micro vibration, caused by local earthquakes, remain coherent at these receivers. After long-time cross correlation, these signals show up around the zero lag. The shift time from zero increases with distance without obvious constant apparent velocity. These strong signals have a broad spectrum varying from $10 \mathrm{~Hz}$ to $80 \mathrm{~Hz}$, indicating complex origins of noise contributed to these signals.

Surface wave signals are absent in our NCFs although clear surface wave excited by our active source can be observed at these receivers. Instead, weak $P$ waves can be observed in NCFs. For receiver pairs whose distance is larger than $150 \mathrm{~m}, \mathrm{P}$ wave signal is easy to identify. But for those pairs whose distances are shorter than $100 \mathrm{~m}$, this signal is not obvious. As mentioned above, there exist large amplitude signals around zero lag. For short-distance pair, the travel time of $\mathrm{P}$ wave is small, so the $\mathrm{P}$ wave may be buried in these strong signals. Considering the existence of high frequency surface wave excited by the active source, the absence of surface wave in NCFs is not due to attenuation. The possible explanation may be in such high frequency, the noise field consists more of body waves while lacks surface waves.

Extracting body waves from noise cross correlation remains challenging, as well as locating source of body waves in ambient noise. Landès et al. (2010) studied the $\mathrm{P}$ waves from noise cross correlation in secondary microseism band and suggested these $\mathrm{P}$ waves were composed of teleseismic $\mathrm{P}$ waves. The source is related to oceanic waves located in deep part of ocean or in the near-coastal regions or both. Similar results are obtained by Koper et al. (2010). The $\mathrm{P}$ wave obtained by Landès et al. (2010) has near vertical incidence and high appar- 
ent velocity larger than $10 \mathrm{~km} / \mathrm{s}$. The weak $\mathrm{P}$ wave observed in our result has a much lower apparent velocity and higher frequency, the origin of this $\mathrm{P}$ wave needs further research.

In this paper, long time ambient noise cross correlation was performed using records from a small aperture array. For the frequencies from $30 \mathrm{~Hz}$ to $70 \mathrm{~Hz}$, there are no obvious surface waves in the NCFs, suggesting absence of surface wave in high-frequency noise field. Weak P waves are observed instead, which indicates high frequency noise filed may consist more of body waves and lack surface waves.

Generation mechanism of the peaks (Figure 2) between $10-20 \mathrm{~Hz}$ and $30-70 \mathrm{~Hz}$ are not known yet. Bonnefoy-Claudet et al. (2006) attributed high frequency noise $(>10 \mathrm{~Hz})$ to culture origin, but Berger et al. (1988) observed spectral peak from $30 \mathrm{~Hz}$ to $70 \mathrm{~Hz}$ in noise field at Kazakhstan which is sparsely populated and tectonically stable as well as far from coasts. Berger et al. (1988) claimed these high frequency noises were generated by natural source such as wind. They also found that local site amplification and exposed terrain can lead to much stronger noise. The Ningqiang array is situated on soft sediments in a valley, and the surrounding mountains may serve as exposed terrain coupling wind motion into ground motion.

Young et al. (1996) proposed that wind-generated noise is broadband $(10-60 \mathrm{~Hz})$, and culture-generated noise is much weaker in this band. Therefore the peaks in the range of 40-70 $\mathrm{Hz}$ we observed in the Ningqiang array are probably generated by interaction between the mountains and wind. But why body wave component is preferentially excited by the wind-topography interaction is not well understood yet, and the origin of body wave remains to be studied.

Acknowledgements Thanks are given to the people who are involved in the Ningqiang experiment, and also to anonymous reviewers for their suggestion to improve this manuscript. This work was supported by Central Public-interest Scientific Institution Basal Research Fund (No. DQJB09B07) and Knowledge Innovation Program of the Chinese Academy of Sciences under grant No. KZCX2-YW-116-1. It was supported partially by National Natural Science Foundation of China (Nos. 40874095, 40730318 and 41004019) and China Earthquake Administration Special Program Fund (Nos. 200808078 and 200808002). Contribution $\mathrm{No}$ is 10FE3007, Institute of Geophysics, China Earthquake
Administration.

\section{References}

Bensen G D, Ritzwoller M H and Shapiro N M (2008). Broadband ambient noise surface wave tomography across the United States. J Geophys Res 113: B05306.

Bensen G D, Ritzwoller M H, Barmin M P, Levshin A L, Lin F, Moschetti M P, Shapiro N M and Yang Y (2007). Processing seismic ambient noise data to obtain reliable broad-band surface wave dispersion measurements. Geophys J Int 169: 1239-1 260.

Berger J, Eissler H K, Vernon F L, Nersesov I L, Gokhberg M B, Stolyrov O A and Tarasov N T (1988). Studies of highfrequency seismic noise in eastern Kazakhstan. Bull Seismol Soc Am 78: 1744-1 758.

Bonnefoy-Claudet S, Cotton F and Bard P Y (2006). The nature of noise wavefield and its applications for site effects studies: A literature review. Earth Sci Rev 79: 205-227.

Brenguier F, Campillo M, Hadziioannou C, Shapiro N M, Nadeau R M and Larose E (2008a). Postseismic relaxation along the San Andreas Fault at Parkfield from continuous seismological observations. Science 12: 1 478-1 481.

Brenguier F, Shapiro N M, Campillo M, Ferrazzini V, Duputel Z, Coutant $\mathrm{O}$ and Nercessian A (2008b). Towards forecasting olcanic eruptions using seismic noise. Nature Geosci 1: 126-130.

Derode A, Larose E, Tanter M, de Rosny J, Tourim A, Campillo $M$ and Fink M (2003). Recovering the Green's function from field-field correlations in an open scattering medium. J Acoust Soc Am 113: 2 973-2 976.

Draganov D, Wapenaar K, Mulder W, Singer J and Verdel A (2007). Retrieval of reflections from seismic background-noise measurements. Geophys Res Lett 34: L04305.

Duputel Z, Ferrazzini V, Brenguier F, Shapiro N, Campillo M and Nercessian A (2009). Real time monitoring of relative velocity changes using ambient seismic noise at the Piton de la Fournaise volcano (La Reunion) from January 2006 to June 2007. J Volcanol Geoth Res 184(1-2): 164-173.

Hasselmann K (1963). A statistical analysis of the generation of microseisms. Rev Geophys 1: 177-210.

Huang W C, Ge H K and Yang W (2009). Survey of the shallow structure on the northeast end of Wenchuan earthauke fault zone by artifical seismic. Chinese J Geophys 52(2): 547-552 (in Chinese with English abstract).

Kedar S, Longuet-Higgins M, Webb F, Graham N, Clayton R and Jones $C$ (2008). The origin of deep ocean microseisms in the North Atlantic Ocean. Proc R Soc London, Ser A 464: 777793.

Koper K D, Seats K and Benz H (2010). On the composition of Earth's short-period seismic noise field. Bull Seismol Soc Am 100: 606-617.

Landès M, Hubans F, Shapiro N M, Paul A and Campillo M (2010). Origin of deep ocean microseisms by using teleseismic body waves. $J$ Geophys Res 115: B05302.

Larose E, Derode A, Corennec D, Margerin L and Campillo M (2005). Passive retrieval of Rayleigh waves in disordered 
elastic media. Phys Rev E 72: 046607.

Lin F, Ritzwoller M H, Townend J, Savage M and Bannister S (2007). Ambient noise Rayleigh wave tomography of New Zealand. Geophys J Int 170: 649-666.

Longuet-Higgins M S (1950). A theory on the origin of microseisms. Philos Trans R Soc London 243: 1-35.

Rhie J and Romanowicz B (2004). Excitation of Earth's continuous free oscillations by atmosphere-ocean-seafloor coupling. Nature 431: 552-556.

Rhie J and Romanowicz B (2006). A study of the relation between ocean storms and the Earth's hum. Geochem Geophys Geosyst 7: Q10004.

Roux P, Sabra K G, Gerstoft P, Kuperman W A and Fehler M C (2005). P-waves from cross-correlation of seismic noise. Geophys Res Lett 32: L19303.

Sabra K G, Gerstoft P, Roux P, Kuperman W A and Fehler M C (2005). Surface wave tomography from microseism in southern California. Geophys Res Lett 32: L14311.

Shapiro N M, Campillo M, Stehly L and Ritzwoller M H (2005). High resolution surface wave tomography from ambient seismic noise. Science 307: 1 615-1 618.

Snieder R (2004). Extracting the Green's function from the correlation of coda waves: A derivation based on stationary phase. Phys Rev E 69: 046610.

Stehly L, Campillo M and Shapiro N M (2006). A study of the seismic noise from its long-range correlation properties. $J$ Geophys Res 111: B10306.

Wapenaar K (2004). Retrieving the elastodynamic Green's function of an arbitrary inhomogeneous medium by cross correlation. Phys Rev Lett 93: 254301.
Weaver R L and Lobkis O I (2001). On the emergence of the Green's function in the correlations of a diffuse field. $J$ Acoust Soc Am 110: 3 011-3 017.

Weaver R L and Lobkis O I (2004). Diffuse fields in open systems and the emergence of the Green's function. $J$ Acoust Soc Am 116: 2731-2 734.

$\mathrm{Xu}$ Z J and Song X D (2009). Temporal changes of surface wave velocity associated with major Sumatra earthquakes from ambient noise correlation. PNAS 106: 14 207-14 212.

Yang Y and Ritzwoller M H (2008). Characteristics of ambient seismic noise as a source for surface wave tomography. Geochem Geophys Geosyst 9: Q02008.

Yang Y, Ritzwoller M H, Levshin A L and Shapiro N M (2007). Ambient noise Rayleigh wave tomography across Europe. Geophys J Int 168: 259-274.

Yao H, van der Hilst R D and de Hoop M V (2006). Surface-wave array tomography in SE Tibet from ambient seismic noise and two-station analysis - I. Phase velocity maps. Geophys J Int 166: 732-744.

Young C J, Chael E P, Withers M M and Aster R C (1996). A comparison of the high-frequency $(>1 \mathrm{~Hz})$ surface and subsurface noise environment at three sites in the United States. Bull Seismol Soc Am 86: 1516-1 528.

Zhan Z W, Ni S D, Helmberger D V and Clayton R W (2010). Retrieval of Moho-reflected shear wave arrivals from ambient seismic noise. Geophys J Int 182(1): 408-420.

Zheng S H, Sun X L, Song X D, Yang Y J and Ritzwoller M H (2008). Surface wave tomography of China from ambient seismic noise correlation. Geochem Geophys Geosyst 9: Q05020, doi:10.1029/2008GC001981. 